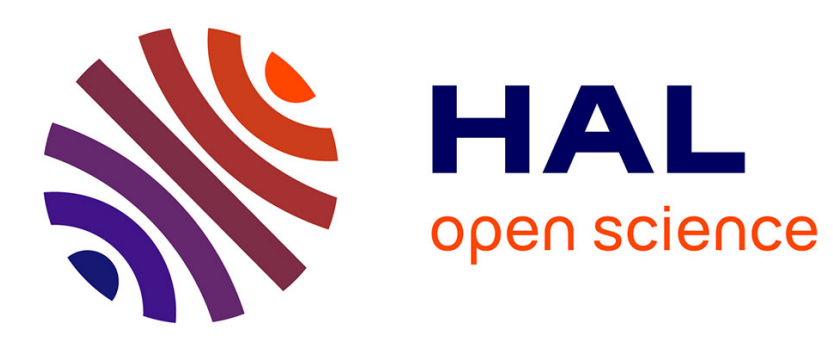

\title{
Méthode de réalisation de couches minces de CdTe par transport gazeux
}

\author{
C. Piaget
}

\section{To cite this version:}

C. Piaget. Méthode de réalisation de couches minces de CdTe par transport gazeux. Revue de Physique Appliquée, 1966, 1 (3), pp.201-203. 10.1051/rphysap:0196600103020100 jpa-00242716

\section{HAL Id: jpa-00242716 https://hal.science/jpa-00242716}

Submitted on 1 Jan 1966

HAL is a multi-disciplinary open access archive for the deposit and dissemination of scientific research documents, whether they are published or not. The documents may come from teaching and research institutions in France or abroad, or from public or private research centers.
L'archive ouverte pluridisciplinaire HAL, est destinée au dépôt et à la diffusion de documents scientifiques de niveau recherche, publiés ou non, émanant des établissements d'enseignement et de recherche français ou étrangers, des laboratoires publics ou privés. 


\title{
MÉTHODE DE RÉALISATION DE COUCHES MINCES DE CdTe PAR TRANSPORT GAZEUX
}

\author{
Par C. PIAGET, \\ Laboratoire d'Électronique et Physique Appliquée, Limeil-Brevannes.
}

\begin{abstract}
Résumé. - Deux méthodes classiques de dépôt de couches minces de CdTe (transport par l'iode et évaporation sous vide) sont transposées des basses pressions à des pressions voisines de l'atmosphère. La dissociation et la cristallisation du CdTe dans un flux d'hydrogène sont étudiées ; différents paramètres et leur rôle sont présentés. On indique les caractéristiques essentielles de ces couches destinées à la réalisation de photopiles solaires.
\end{abstract}

Abstract. - Two well known methods for deposition of CdTe thin layers (iodine transport and vacuum deposition) are altered from low to nearly atmospheric pressure. Dissociation and crystallization of CdTe in hydrogen flow are studied and the effect of the different parameters is given. The properties of these layers used as solar cells are discussed.

Introduction. - Un certain nombre de méthodes de dépôt de couches minces de CdTe a déjà été mis au point. Nous citerons pour mémoire :

- les techniques d'évaporation du composé, dont la plus élaborée est celle mise au point par Elliott [1];

- les techniques d'évaporation des composants, comme celle décrite par D. A. Cusano [2];

- la technique de transport gazeux par voie chimique, utilisée par Zh. I. Alferov et al. [3] utilise, en tube scellé sous vide, les deux réactions :

$$
\begin{aligned}
2 \mathrm{CdTe} & +\mathrm{I}_{2} \\
2 \mathrm{CdI} & \longrightarrow \frac{1}{2} \mathrm{Te}_{2} \rightleftarrows \mathrm{CdI}+\mathrm{Te}_{2}
\end{aligned}
$$

la première correspondant à la dissociation du cristal-source dans la zone de haute température du tube $\left(800^{\circ} \mathrm{C}\right.$ environ), la deuxième à la cristallisation du CdTe dans la zone plus froide $\left(700^{\circ} \mathrm{C}\right)$.

Toutes les techniques citées nécessitent un appareillage relativement compliqué, tel un ensemble évaporateur spécial, ou un grand nombre d'opérations délicates et, de toutes façons, le pompage d'une enceinte jusqu'à des pressions assez basses. Ces considérations nous ont amenés à étudier leur transposition à des appareillages travaillant au voisinage de la pression atmosphérique en milieu neutre ou réducteur.

I. Transport par l'iode. -- Le principe d'une telle méthode repose sur le fait qu'il est possible, lorsqu'un gaz balaye la surface d'un cristal, moyennant des vitesses de déplacement du gaz relativement faibles, de s'approcher suffisamment de l'équilibre solide-vapeur pour que les réactions nécessaires au transport gazeux soient possibles dans des domaines de température permettant la cristallisation du CdTe.

L'appareillage est extrêmement simple : il con- siste en un four tubulaire à trois zones de température, chauffé par effet Joule et alimenté en gaz neutre purifié (argon) :

- La première zone, de température ajustable entre 50 et $200^{\circ} \mathrm{C}$, permet d'établir des pressions partielles d'iode de 3 à $10^{3}$ torr.

- La deuxième zone, réglée à des températures comprises entre $850^{\circ}$ et $1100^{\circ} \mathrm{C}$, reçoit le cristalsource de CdTe.

- La troisième zone, séparée de la précédente par un gradient de température important, est réglée sur $30 \mathrm{~cm}$ à $\pm 5^{\circ} \mathrm{C}$ près à une température de l'ordre de $600^{\circ} \mathrm{C}$; elle est le siège de la cristallisation du CdTe véhiculé par le flux gazeux sous forme de vapeurs de $\mathrm{CdI}$ et $\mathrm{Te}_{2}$.

Les résultats obtenus montrent qu'un tel transport est possible, mais les difficultés rencontrées pour parvenir à des couches homogènes et la mauvaise tenue de la silice comme matériau de four nous ont amenés à étudier une autre transposition ; l'évaporation-condensation en milieu réducteur.

2. Transport par l'hydrogène. - L L'étude thermodynamique de l'équilibre

$$
\mathrm{Cd} \mathrm{Te}_{(\mathrm{s})} \rightleftarrows \mathrm{Cd}_{(\mathrm{g})}+\frac{1}{2} \mathrm{Te}_{2(\mathrm{~g})}
$$

montre que les pressions de vapeur de $\mathrm{Cd}$ et $\mathrm{Te}_{2}$ (fig. 1) sur le CdTe sont dans un rapport 2, ce qui implique une évaporation du CdTe sans écart à la stoechiométrie.

Supposons un élément de volume d'hydrogène balayant un cristal de CdTe à une température $T_{1}$, avec une vitesse telle que l'équilibre solide-vapeur soit atteint, puis porté à une température plus faible $T_{2}$ : les pressions partielles de $\mathrm{Cd}$ et $\mathrm{Te}_{2}$ établies sur le CdTe à $T_{1}$ étant supérieures aux 


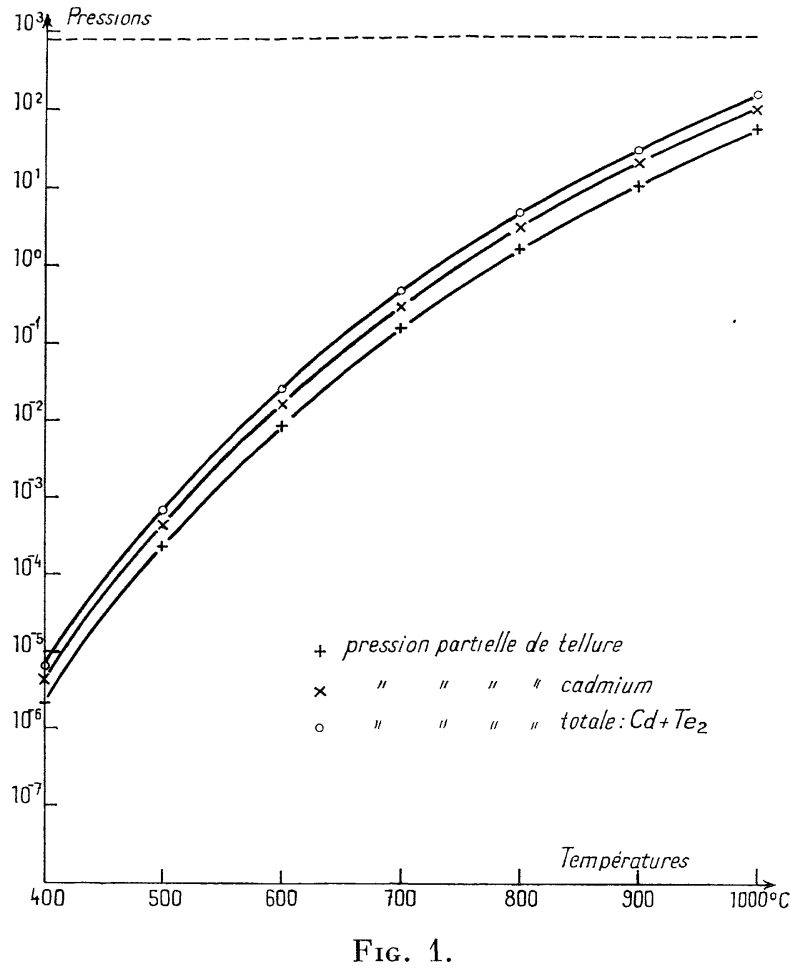

pressions d'équilibre à $T_{2}$, il y a condensation des vapeurs sous forme de CdTe car

$$
k_{\mathrm{Cd}} \# k_{\mathrm{Te}_{\mathbf{2}}} \gg k_{\mathrm{Cd}} \mathrm{Te} \text {. }
$$

Le dispositif permettant de concréiiser un tel ensemble de phénomènes (fig. 2) est un four tubu-

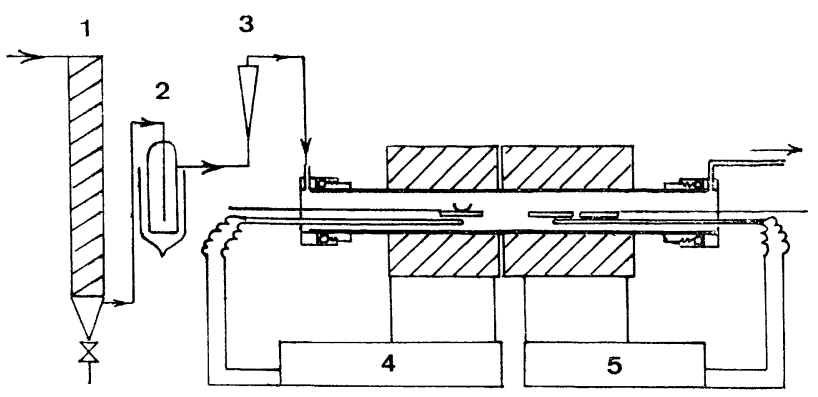

Fig. 2. - 1. Colonne de charbon actif, - 2. Piège à azote liciuide. - 3. Débitmètre. - 4 el 5 . Régulation de la température.

laire en alumine à deux zones de température, alimenté en azote et hydrogène purifié. Le chauffage, par effet Joule, est régulé à $\pm 5^{\circ} \mathrm{C}$, le profil de température est indiqué sur la figure 3 .

$\mathrm{La}$ structure et l'aspect des couches obtenues dépendent directement des variations locales et de la valeur absolue de la température et du débit d'hydrogène. Nous allons étudier succinctement l'influence de ces divers paramètres.

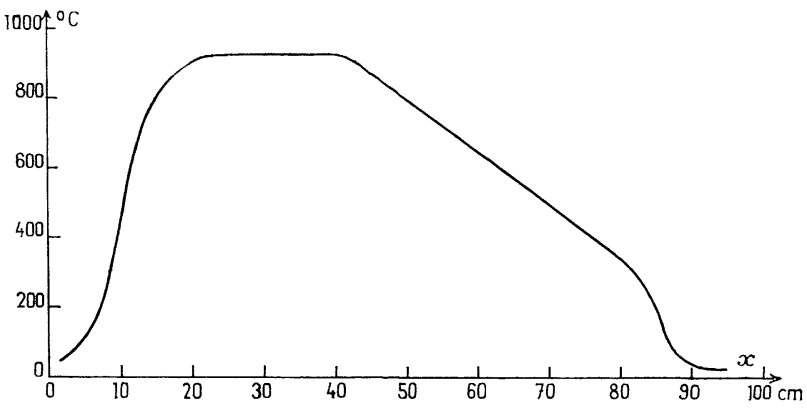

FIG. 3. - Profil de température du four à transport.

Le profil de température. - La vitesse de cruissance des couches augmente avec la valeur absolue du gradient de température, elle est nulle pour des gradients nuls ou positifs (le sens positif des $x$ est celui du déplacement d'hydrogène).

Les valeurs absolues des températures. - Il n'y a de cristallisation possible que pour des températures de support comprises dans l'intervalle $\left(700-500^{\circ} \mathrm{C}\right)$.

La température de la source peut varier dans un grand intervalle $\left(800-1090{ }^{\circ} \mathrm{C}\right)$, cependant lorsqu'elle dépasse $950^{\circ} \mathrm{C}$ non seulement les vitesses d'évaporation deviennent grandes mais la cristallisation des couches, au lieu d'être isotrope, se fait en aiguilles. Il semble en effet que les pressions partielles de cadmium et tellure, au début de la région de gradient, soient telles que la croissance ne soit plus limitée par la fréquence des chocs sur le cristal, mais par la mobilité des atomes à la surface du CdTe, différente suivant les plans cristallographiques, ce qui entrâne une croissance anisotrope des couches.

La ritesse des gaz. - Elle doit être déterminée pour chaque profil de température: suffisamment grande pour éviter que les turbulences jouent un rôle prépondérant dans la propagation des vapeurs, suffisamment faible pour que toutes les vapeurs puissent se conderser dans la zone de cristallisation.

Mécanisme du transport. - Au niveau du cristal-source, il s'établit une pression partielle de $\mathrm{Cd}$ et $\mathrm{Te}_{2}: p\left(x_{0}\right)$; un peu inférieure à la pression partielle d'équilibre : $p_{\text {équi }}\left(x_{0}\right)$; à cause du gradient de température $p(x)$ devient supérieur à $p_{\text {équi }}(x)$ en un point $x_{1}$ à partir duquel les vapeurs commencent à se condenser.

Si l'on élimine le cristal-source, la couche déposée en $x_{1}$ joue le rôle de source secondaire, elle se réévapore et le CdTe déposé à plus basses températures continue à croître.

Caractéristiques des couches déposées. Les couches déposées sur des supports amorphes ou 
polycristallins sont toutes polycristallines avec des diamètres moyens de grains :

$$
\begin{aligned}
& \text { - de } 5 \mu \mathrm{m} \text { à } 550^{\circ} \mathrm{C} \text {, } \\
& \text { - de } 15 \mu \mathrm{m} \text { à } 650^{\circ} \mathrm{C} \text {, } \\
& \text { - de } 70 \mu \mathrm{m} \text { à } 700^{\circ} \mathrm{C} \text {. }
\end{aligned}
$$

La figure 4 montre la répartition spectrale du facteur de transmission optique d'une couche de $0,8 \mu \mathrm{m}$ d'épaisseur.

Le dopage $n$ des couches étant réalisé par introduction d'indium à la même température que la source de CdTe les résistivités obtenues couramment sont de quelques $\mathrm{k} \Omega . \mathrm{cm}$, certainement améliorables puisqu'il a été obtenu des valeurs de quelques $\Omega \mathrm{cm}$. Il a été réalisé quelques photopiles par évaporation de $\mathrm{Cu}_{2} \mathrm{Te}$ et d'une grille d'or sur de telles couches déposées directement sur molybdène, dont les rendements, de l'ordre de $1 \%$, sont limités par des résistances-série très importantes dues à la résistivité du CdTe et au contact CdTe-molybdène très résistant et redresseur.

Conclusion. - Il apparaît possible par une telle méthode de réaliser industriellement, et avec un bas

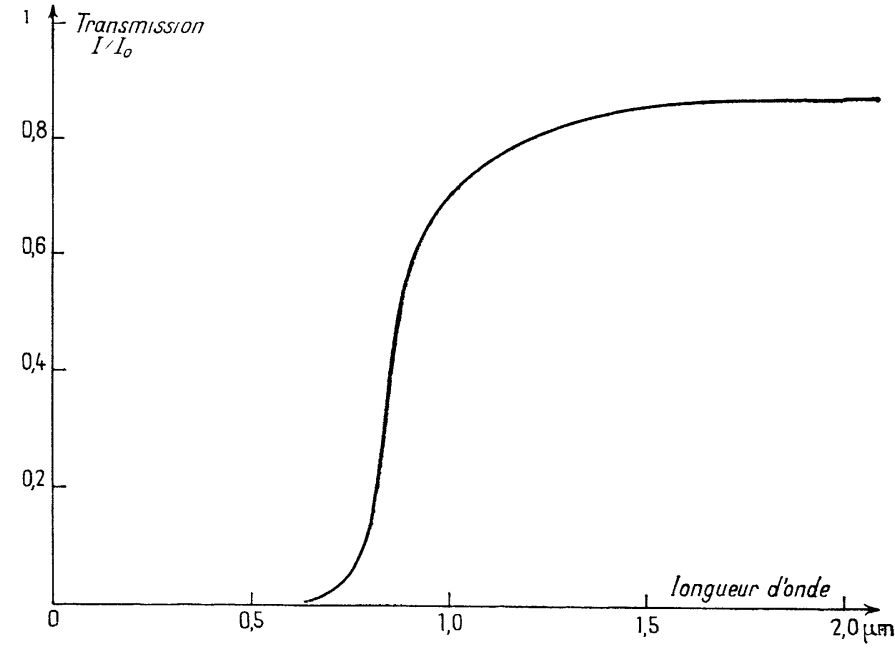

FIg. 4.

prix de revient, des photopiles au CdTe en lui adjoignant, pour la réalisation de la couche, un procédé du type décrit par D. A. Cusano (réf. [2]).

\section{BIBLIOGRAPHIE}

[1] Elliott (J. F.), Halsted (R. E.) et Coghill (H. D.), 15th Annual Proceedings Power Source Conference, 9, 10, 11, 1951 May.
[2] Cusano (D. A.), Solid State Electronics, 1963, 6, no 3, 217.

[3] Alferov, (Zh. I.), Soviet Physics-Solid State, février $1965,6,1865$. 